\title{
Nova abordagem para identificar conexões disciplinares usando mapas conceituais: em busca da interdisciplinaridade no Ensino Superior*
}

\section{New approach to identify disciplinary connections using concept maps: in pursuit of interdisciplinarity in Higher Education}

\author{
Paulo Rogério Miranda Correia ${ }^{1}$. Gislaine Banchetti Cordeiro ${ }^{1}$. \\ Camila Aparecida Tolentino Cicuto $^{2}$ • Patrícia Grandino Junqueira ${ }^{1}$
}

\begin{abstract}
Resumo: A interdisciplinaridade pode ser considerada uma maneira diferente de enfrentar o impacto da explosão do conhecimento científico no âmbito educacional. Apesar da sua importância, as ações interdisciplinares nas salas de aula ainda são pouco frequentes, visto as dificuldades de planejamento e implementação enfrentadas pelos docentes. A literatura não apresenta um procedimento robusto para ajudar os professores a identificar possíveis conexões entre os seus cursos. O presente trabalho explorou o mapeamento conceitual (MC) como forma de identificar pontos de acoplamento entre as disciplinas Ciências da Natureza e Psicologia, Educação e Temas Contemporâneos. Após quatro etapas de elaboração e revisão dos MCs, foi possível representar a interface entre essas disciplinas e identificar os conceitos tecnologia, complexidade e religião como elementos promissores para estimular a interdisciplinaridade. A utilização dos procedimentos descritos neste trabalho pode ser útil para se buscar a interdisciplinaridade em outras disciplinas de diferentes níveis da educação formal.
\end{abstract}

Palavras-chave: Ensino Superior. Currículo. Interdisciplinaridade. Mapa conceitual.

Abstract: Interdisciplinarity can be considered a different way to address the impact of the scientific knowledge explosion in education. Despite its importance, interdisciplinary activities in classrooms are rare due to planning and implementation difficulties faced by instructors. The literature does not offer a robust procedure to support instructors to identify possible connections between their courses. The present study explored concept mapping as a way to identify connections between two courses about Natural Sciences and Psychology, Education and Contemporary Issues. After four steps for developing and revising concept maps, it was possible to represent the interface between these courses, and identify the concepts technology, complexity and religion as the promising ones to foster interdisciplinarity. The procedures described are useful to characterize interdisciplinary connections among courses of any level of formal education.

Keywords: Higher education. Curriculum. Interdisciplinarity. Concept map.

\footnotetext{
* Parte desse trabalho foi apresentado oralmente durante o VIII Encontro Nacional de Pesquisa em Educação em Ciências (ENPEC) e I Congreso Iberoamericano de Investigación en Enseñanza de las Ciéncias (CIEC), realizado na Universidade Estadual de Campinas entre os dias 05 e 09 de dezembro de 2011.

${ }^{1}$ Escola de Artes, Ciências e Humanidades (EACH), Universidade de São Paulo (USP), Avenida Arlindo Bettio, 1000, Bloco I, sala 320D, Ermelino Matarazzo, CEP 03828-000, São Paulo, SP, Brasil. E-mail: prmc@usp.br

${ }^{2}$ Programa de Pós-Graduação Interunidades em Ensino de Ciências, Universidade de São Paulo (USP), São Paulo, SP, Brasil.
} 


\section{Introdução}

A explosão do conhecimento científico e os paradigmas da sociedade do conhecimento trouxeram novos desafios à escola, que não são respondidos adequadamente utilizando-se a configuração tradicional da sala de aula (UNESCO, 2005). A leitura integrada dos conteúdos de diferentes disciplinas é um aspecto fundamental que deve ser considerado na formação oferecida pelos cursos de graduação, a fim de se prepararem profissionais para lidar com a crescente complexidade da sociedade do conhecimento (FORD; FORMAN, 2006; MORAN, 2002). A interdisciplinaridade, muitas vezes, é definida como a integração entre disciplinas (KLEIN, 1996; LATTUCA, VOIGT; FATH, 2004). Nesse contexto, a abordagem interdisciplinar deve ser explorada de forma planejada, e o currículo deve facilitar a inter-relação entre os conteúdos disciplinares (LAMBERT, TERENZINI; LATTUCA, 2007; LATTUCA; STARK, 1994; STARK et al., 1997). Apesar da sua importância, as ações interdisciplinares nas salas de aula ainda são pouco frequentes, visto as dificuldades de planejamento e implementação enfrentadas pelos docentes.

A organização do currículo escolar em disciplinas foi a saída encontrada para lidar com a enorme quantidade de informação acumulada pela humanidade, facilitando e padronizando a transmissão do conhecimento especializado. Por esse motivo, as disciplinas possuem um papel hegemônico na elaboração e organização do currículo escolar (LATTUCA, 2001; LATTUCA, 2002). A fragmentação do conhecimento em disciplinas é empregada com a finalidade de organizar e sistematizar os saberes necessários à formação dos alunos. Apesar de todas as tentativas de organização de um currículo não disciplinar, é por meio de disciplinas que o conhecimento é transmitido em grande parte das instituições de ensino (KLEIN, 1996).

Em comparação com as práticas disciplinares tradicionais, a interdisciplinaridade pode ser considerada uma maneira diferente de enfrentar o impacto da explosão do conhecimento científico no âmbito educacional, admitindo a organização dos conteúdos em função de temas contemporâneos que exigem a articulação dos saberes de vários campos do conhecimento (KLEIN 1996, LATTUCA, 2001; WEINGART; STEHR, 2000). Além disso, a interdisciplinaridade valoriza a inovação, a criatividade, e rompe com o paradigma da padronização, aproximando um pouco mais a educação formal das novas demandas da sociedade do conhecimento (BAKER; LATTUCA, 2010; SAWYER, 2006).

A revisão da literatura relacionada com a organização interdisciplinar de currículos do Ensino Superior revela uma lacuna que dificulta o estabelecimento de vínculos entre os conteúdos disciplinares. Não há um procedimento robusto capaz de auxiliar os docentes a identificarem possíveis conexões entre as disciplinas presentes no currículo de um curso de graduação. A experiência do nosso grupo de pesquisa com o mapeamento conceitual indica que essa técnica pode ser utilizada para representar graficamente os conteúdos disciplinares, a fim de facilitar o processo de construção colaborativa de conhecimento (CRANDALL, KLEIN; HOFFMAN, 2006; FISCHER et al., 2002). Os tópicos a seguir explicam melhor como isso pode ser feito, considerando-se a participação: de especialistas em mapeamento conceitual (P.R.M.C. e C.A.T.C.), dos docentes responsáveis por disciplinas de graduação (P.G.J. e P.R.M.C.) e de uma aluna de graduação (G.B.C.), que faz o papel de mediação entre os participantes mencionados, uma vez que ela já cursou as disciplinas e compreende a técnica de mapeamento conceitual. 


\section{Mapas conceituais para gestão da informação e do conhecimento}

O mapa conceitual (MC) é uma representação gráfica de conceitos e suas inter-relações. Ele possui quatro elementos característicos (CORREIA, INFANTE-MALACHIAS; GODOY, 2008) que o distinguem dos demais organizadores gráficos de informação e conhecimento. A Figura 1 apresenta, de forma esquemática, esses elementos: proposição (P), pergunta focal $(\mathrm{PF})$, hierarquia $(\mathrm{H})$ e revisão continuada $(\mathrm{RC})$.

Figura 1. Os quatro elementos que diferenciam os MCs dos demais tipos de organizadores gráficos: as proposições $(P)$, a pergunta focal (PF), a hierarquia $(H)$ e a revisão continuada (RC).

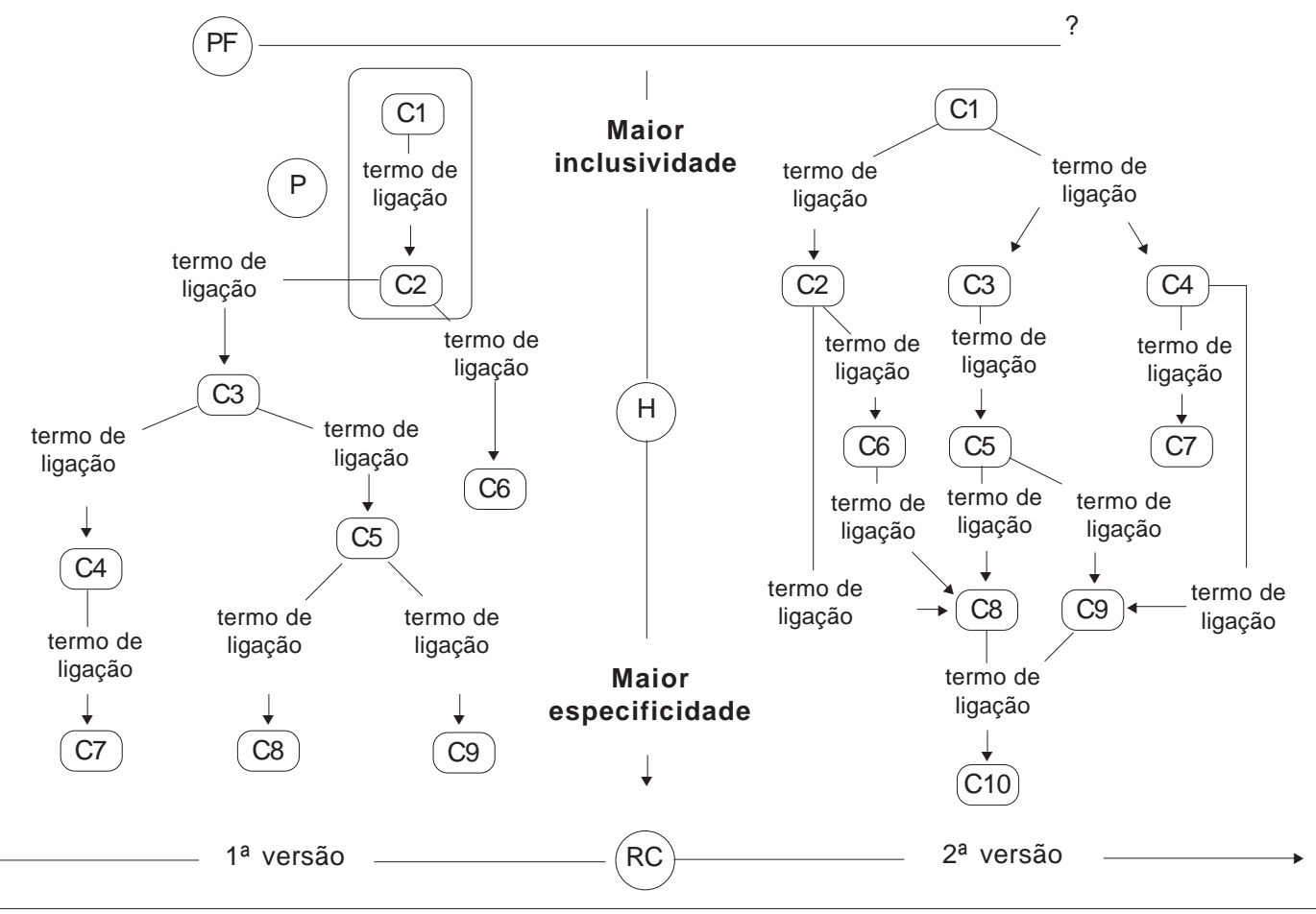

Fonte: Elaborada pelos autores

Os MCs são formados por um conjunto de conceitos imersos em uma rede de proposições (NOVAK, 2010). As proposições (P), por sua vez, são formadas por um conceito inicial, um conceito final e um termo de ligação que expressa, claramente, qual a relação conceitual existente (Figura 1). Uma seta indica o sentido de leitura para que a proposição tenha clareza semântica. A obrigatoriedade de incluir o termo de ligação torna os MCs mais poderosos do que outros organizadores gráficos pelo fato de ele reduzir as idiossincrasias da representação do conhecimento. Essa característica torna o mapeamento conceitual uma técnica atraente para favorecer processos colaborativos, onde os participantes precisam comunicar seus conhecimentos e ideias da forma mais clara possível (CORREIA, 2012; MOON et al., 2011; TORRES; MARRIOT'T, 2009). Isso não se verifica com outros esquemas, tais como 
os mapas mentais, que sempre precisam ter seu conteúdo explicado pelos seus autores para serem compreendidos (DAVIES, 2011).

A pergunta focal (PF) é o parâmetro utilizado para seleção dos conceitos e dos termos de ligação mais relevantes, durante o processo de elaboração do MC. A PF funciona como "guia" para que os mapeadores sejam criteriosos na elaboração de proposições que expressem relações pertinentes com a pergunta proposta. Isso evita a elaboração de MCs contendo somente generalidades, ou um número excessivo de conceitos. Em ambos os casos, a comunicação de ideias é prejudicada pela falta de foco.

A hierarquia $(\mathrm{H})$ é o ajuste fino da estrutura da rede proposicional do MC. A organização dos conceitos, em função do seu caráter mais inclusivo (no topo do MC), ou mais específico (na parte inferior do MC), favorece a compreensão das relações estabelecidas no MC. Por meio da hierarquia, é possível inferir como o mapeador expressa seu conhecimento: com processos de diferenciação progressiva (ver $1^{\mathrm{a}}$ versão na Figura 1) e/ou de reconciliação integrativa (ver $2^{a}$ versão na Figura 1). Cabe ressaltar que estes processos refletem a estrutura de conhecimento do mapeador e a forma pela qual ele identifica as diferenças e similaridades entre conceitos ao estabelecerem proposições (NOVAK, 2010).

A revisão continuada (RC) refere-se à natureza dinâmica dos MCs. Esta característica contribui para a contínua melhoria do conteúdo expresso nos MCs. Diferente do que acontece com exercícios e provas, a busca pela "resposta certa" faz pouco sentido quando utilizamos MCs. A possibilidade de revê-los várias vezes ao longo do processo confere aos MCs o seu caráter dinâmico. $\mathrm{Na}$ Figura 1 esta característica foi destacada com uma seta horizontal na parte inferior dos MCs. Esta representação tem como objetivo destacar que o MC inicial (1 ${ }^{\mathrm{a}}$ versão) foi aperfeiçoado, gerando um novo MC ( $2^{a}$ versão).

Os quatro elementos que diferenciam os MCs dos demais tipos de organizadores gráficos (P, PF, H e RC) possibilitam que eles sejam utilizados como instrumento de gestão da informação e do conhecimento, por meio de processos colaborativos. Espera-se, assim, que este trabalho estimule a integração de conteúdos disciplinares através da colaboração entre docentes de áreas de conhecimento diferentes utilizando MCs.

\section{Construção colaborativa do conhecimento}

Atividades colaborativas envolvem a interação entre vários participantes. A colaboração deve ser compreendida como algo que extrapola a mera contribuição isolada de cada indivíduo. Os participantes envolvidos no processo de colaboração necessitam de algum grau de compreensão mútua para a efetiva realização das atividades (BAKER et al., 1999), a fim de que seja possível obter sinergia. O MC viabiliza a colaboração porque favorece a construção colaborativa do conhecimento (CCC), que pode ser descrita em três etapas: (1) externalização, (2) elicitação e (3) busca por consenso (Figura 2). A última etapa pode ocorrer por meio de conflitos (3.1) ou por integração (3.2), dependendo do nível de interação e sinergia entre os participantes (FISCHER et al., 2002).

O mapeamento conceitual pode auxiliar na (1) externalização dos modelos mentais idiossincráticos dos indivíduos. Esta etapa da CCC (Figura 2) é favorecida quando os MCs são construídos individualmente, já que a externalização requer a caracterização dos conheci- 
Figura 2. Principais etapas para descrever a construção colaborativa de conhecimento (CCC): 1. externalização (elaboração individual de MCs), 2. elicitação e 3. construção de consenso por conflito ou integração (elaboração colaborativa de MCs).

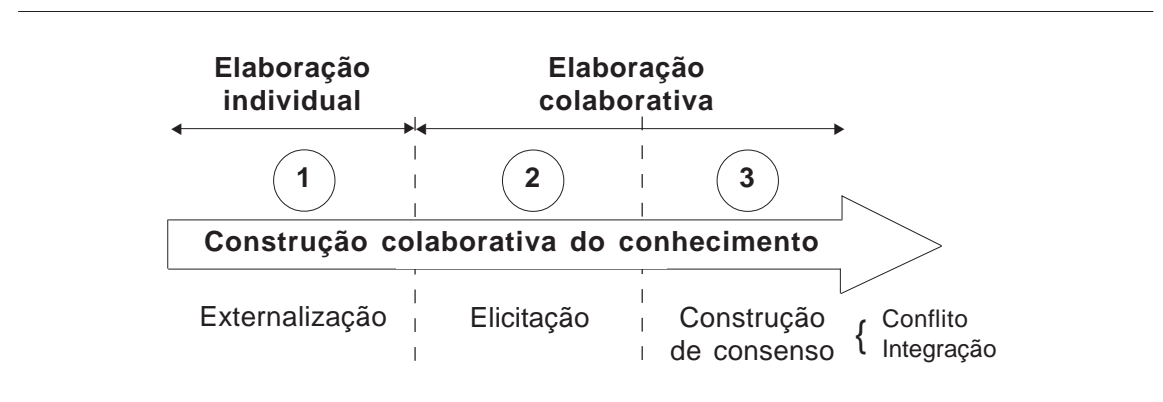

Fonte: Elaborada pelos autores

mentos prévios de todos os participantes. A partir da externalização, é possível trocar significados idiossincráticos sobre conceitos, além de fazer o diagnóstico e a correção de concepções equivocadas dos participantes durante o processo de construção colaborativa do conhecimento (FISCHER et al., 2002).

As etapas que contemplam a (2) elicitação e (3) construção de consenso são favorecidas pelos MCs colaborativos (Figura 2). O mapeamento conceitual pode auxiliar na elicitação dos modelos mentais dos indivíduos. Isso facilita a negociação de significados, visto que os conhecimentos prévios dos integrantes do grupo podem ser identificados com mais clareza e rapidez. É na etapa de elicitação que os indivíduos organizam e explicitam os seus conhecimentos relevantes para a consecução de uma tarefa. A elicitação frequentemente ocorre durante uma dinâmica discursiva que envolve perguntas e respostas, contribuindo para o esclarecimento e a revisão dos conhecimentos prévios. Essa etapa é parcialmente responsável pelo sucesso nesse processo colaborativo (FISCHER et al., 2002).

Os MCs colaborativos também favorecem a negociação de significados, viabilizando o exercício de síntese e de revisão pelos integrantes do grupo durante a construção de consenso. A construção de consenso por conflito (3.1) destaca-se por: (a) soluções coletivas dos conhecimentos relacionados com a tarefa; (b) conflitos de ideias na busca pelo consenso; e (c) consenso efetivo dos integrantes ao gerar o conflito. Já a construção de consenso por integração (3.2) ocorre por meio de: (a) soluções individuais dos conhecimentos relacionados com a tarefa; (b) integração de ideias individuais numa interpretação comum, e (c) consenso ilusório dos integrantes ao evitarem o conflito (FISCHER et al., 2002).

O mapeamento conceitual colaborativo apresenta um grande potencial para elicitar o conhecimento de especialistas (docentes das disciplinas) e favorecer a negociação de significados por intermédio da construção de consenso. Essa é uma utilização sofisticada dos MCs, e seu sucesso depende da habilidade do entrevistador/mediador em saber utilizar o mapeamento conceitual para formular perguntas visando à explicitação do conhecimento especializado e a organização/modelagem da informação na forma de MCs (CRANDALL, KLEIN; HOFFMAN, 2006). No caso particular desse trabalho, os especialistas (docentes) podem se beneficiar do uso de MCs para avaliar a existência (ou não) de vínculos interdisciplinares entre suas disciplinas. 


\section{Objetivo}

O presente trabalho tem como objetivo propor um procedimento de elaboração e análise de mapas conceituais para identificar a existência (ou não) de conexões disciplinares. Os MCs foram utilizados como forma de estimular a integração de disciplinas em busca de relações interdisciplinares. Além disso, os primeiros resultados são apresentados para ilustrar o potencial dessa abordagem, a partir do MC elaborado para Ciências da Natureza (CN) e Psicologia, Educação e Temas Contemporâneos (PET), oferecidas na Escola de Artes, Ciências e Humanidades (EACH/USP Leste).

\section{Procedimentos de pesquisa}

\section{Contexto da pesquisa: Ciclo Básico da USP Leste}

A EACH/USP Leste é a primeira unidade da Universidade de São Paulo sem departamentos. Ela apresenta um Ciclo Básico que reúne um conjunto de atividades que são cumpridas por todos os mil e vinte ingressantes ao longo do $1^{\circ}$ ano da graduação. Seis disciplinas de caráter geral (duas horas semanais) são oferecidas no Ciclo Básico para contribuir com a formação geral dos alunos (Quadro 1). Isso é uma clara evidência de que a não-fragmentação do conhecimento e a interdisciplinaridade são pressupostos que devem ser assumidos durante o desenvolvimento das atividades acadêmicas de ensino, pesquisa e extensão (GOMES, 2005; CORREIA et al., 2010).

Para este estudo, foram selecionadas as disciplinas Ciências da Natureza (CN) e Psicologia, Educação e Temas Contemporâneos (PET), ministradas nos $1^{\circ}$ e $2^{\circ}$ semestres letivos, respectivamente. A disciplina $\mathrm{CN}$ contempla elementos da cultura das ciências naturais, enquanto PET discute conteúdos vinculados à cultura das ciências humanas e sociais. A seleção das disciplinas baseou-se na dificuldade em se estabelecer um diálogo entre disciplinas localizadas nessas duas culturas, conforme descreve C. P. Snow (1995).

Quadro 1. Disciplinas gerais que compõem o Ciclo Básico da EACH/USP Leste. Em destaque, as disciplinas selecionadas para o estudo, Ciências da Natureza e Psicologia, Educação e Temas Contemporâneos.

\begin{tabular}{|l|c|c|}
\hline \multicolumn{1}{|c|}{ Disciplinas } & $\mathbf{1}^{\circ}$ Semestre & $\mathbf{2}^{\circ}$ Semestre \\
\hline ACH0011 Ciências da Natureza* & $X X X$ & \\
\hline ACH0021 Tratamento e Análise de Dados/Informações & $X X X$ & \\
\hline ACH0031 Sociedade, Multiculturalismo e Direitos & $X X X$ & $X X X$ \\
\hline ACH0012 Psicologia, Educação e Temas Contemporâneos* & & $X X X$ \\
\hline ACH0022 Sociedade, Meio Ambiente e Cidadania & & $X X X$ \\
\hline ACH0032 Arte, Literatura e Cultura no Brasil & & \\
\hline
\end{tabular}

*Disciplinas selecionadas para a realização desse trabalho.

Fonte: Elaborado pelos autores 


\section{Elaboração dos mapas conceituais: procedimento para estimular integrações disciplinares}

O procedimento apresentado para elaboração dos MCs foi desenvolvido com o objetivo de estimular a integração de conceitos provenientes de disciplinas diferentes. Os MCs foram utilizados para representar os conteúdos abordados pelas disciplinas A (CN) e B (PET) com elevado grau de precisão. Para isso, eles foram elaborados através das quatro etapas descritas no Quadro 2.

Quadro 2. Procedimento para elaboração e revisão de MCs utilizados para representar os conteúdos abordados pelas disciplinas.

\begin{tabular}{|c|l|l|c|}
\hline Etapa & \multicolumn{1}{|c|}{ Objetivo } & \multicolumn{1}{|c|}{ Descrição } & MC \\
\hline 1 & $\begin{array}{l}\text { Impressões da } \\
\text { aluna }\end{array}$ & $\begin{array}{l}\text { A aluna matriculada no 3o ano do curso de Licenciatura em } \\
\text { Ciências da Natureza elabora um MC para expressar como ela } \\
\text { percebe as relações conceituais da disciplina. }\end{array}$ & MC-1 \\
\hline 2 & $\begin{array}{l}\text { Revisão da } \\
\text { clareza semântica }\end{array}$ & $\begin{array}{l}\text { O MC-1, elaborado pela aluna, é apresentado para os especialistas } \\
\text { em mapeamento conceitual. O objetivo é garantir a clareza } \\
\text { semântica das proposições, a fim de potencializar o uso do MC-1 } \\
\text { no processo de construção colaborativa de conhecimento (CCC). } \\
\text { Correções conceituais somente podem ser feitas pela aluna, que já } \\
\text { cursou o Ciclo Básico. }\end{array}$ & MC-2 \\
\hline 3 & $\begin{array}{l}\text { Impressões do } \\
\text { docente }\end{array}$ & $\begin{array}{l}\text { O MC-2, com elevado grau de clareza semântica, é apresentado ao } \\
\text { docente responsável pela disciplina em uma entrevista. Todos os } \\
\text { questionamentos são oriundos da leitura conjunta do MC-2, e as } \\
\text { respostas são consideradas para revisar a versão atual do MC da } \\
\text { disciplina. O objetivo é incluir o ponto de vista do especialista no } \\
\text { conteúdo da disciplina, por meio da exclusão, inclusão e alteração } \\
\text { de conceitos e proposições. }\end{array}$ & MC-3 \\
\hline 4 & $\begin{array}{l}\text { Revisão da } \\
\text { clareza semântica } \\
\text { e do nível de } \\
\text { detalhamento }\end{array}$ & $\begin{array}{l}\text { O MC-3 é apresentado aos especialistas em mapeamento } \\
\text { conceitual. O objetivo dessa etapa é verificar a clareza semântica } \\
\text { das proposições e avaliar o nível de detalhamento do conteúdo da } \\
\text { disciplina. Esse requisito é importante para viabilizar a comparação } \\
\text { de MC-4 proveniente de várias disciplinas: esses mapas } \\
\text { conceituais devem possuir um número similar de conceitos e } \\
\text { proposições para que nenhuma delas seja favorecida na busca } \\
\text { por conexões disciplinares (MC-5). }\end{array}$ & MC-4 \\
\hline
\end{tabular}

Fonte: Elaborado pelos autores

As Etapas 1-4 são fundamentais para a identificação de conexões entre as disciplinas. Dois MC-4 (Figura 3) foram apresentados aos docentes para a realização de uma entrevista em que todos os questionamentos foram oriundos da leitura conjunta dos MC-4; e as respostas viabilizaram o desenvolvimento de relações entre esses MCs. O objetivo foi explicitar as conexões entre os conteúdos disciplinares, por meio da exclusão, inclusão e alteração de conceitos e proposições.

A Figura 3 mostra o papel da preparação e as etapas de revisão (Quadro 2) para a colaboração. O objetivo foi mesclar o MC-4 de cada disciplina, ligando os conceitos deles por meio de algumas perguntas e respostas. 
Figura 3. Representação esquemática dos mapas conceituais obtidos para duas disciplinas (A e B), visando à elaboração de um MC que represente as possíveis conexões conceituais entre elas (MC-5).

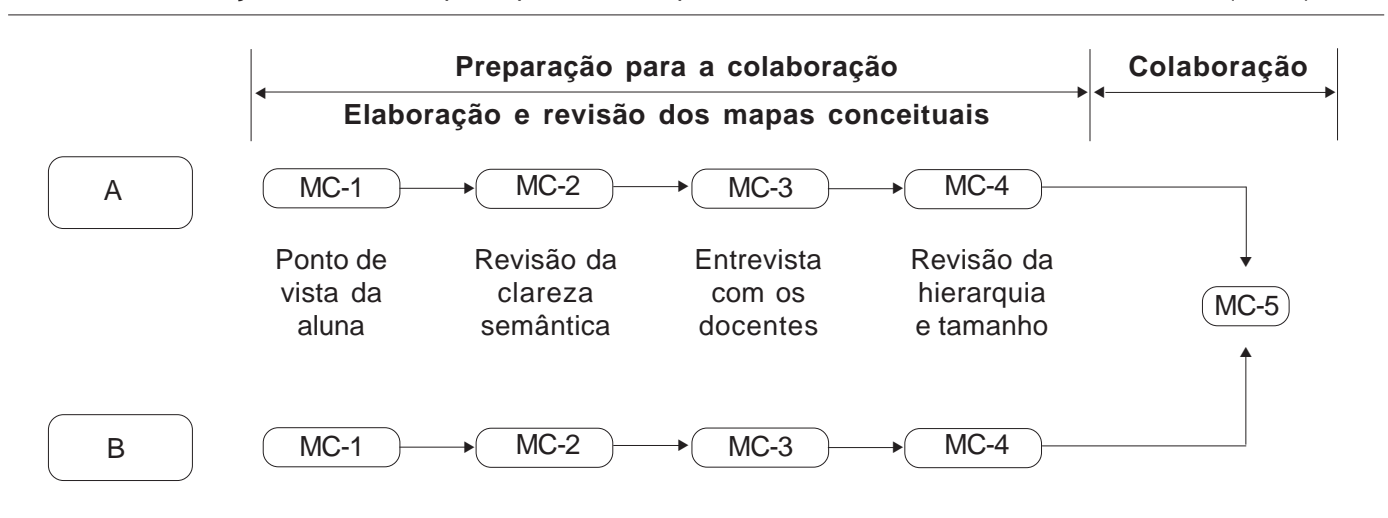

Fonte: Elaborada pelos autores

\section{Análise da interface proposicional interdisciplinar: procedimento para identificar integrações disciplinares}

O MC-5 é considerado como o dado de pesquisa mais relevante para avaliar e classificar as conexões entre as disciplinas. Isso é feito por meio da identificação da interface proposicional interdisciplinar (IPID), que é a região que melhor pode descrever as interações entre os conteúdos específicos de cada disciplina (Figura 4).

Os conceitos e as proposições da IPID são utilizados para caracterizar as conexões estabelecidas entre as disciplinas. A expectativa foi apresentar novos subsídios para a reflexão dos docentes que dominam os conhecimentos específicos das disciplinas, a fim de estimular a busca por relações interdisciplinares latentes e inexploradas no currículo.

Os conceitos C1-C3 (Figura 4) são os eixos organizadores da disciplina A que ajudam a identificar possíveis vinculações com o conteúdo programático da disciplina B. Os conceitos que representam os eixos organizadores da B são C9-C11. Na interface entre as disciplinas (IPID) é possível identificar os conceitos C5-C8 como pontos mais favoráveis para o estabelecimento de vinculações interdisciplinares. O conceito $\mathrm{C} 4$ não foi classificado como eixo organizador, nem como conceito da IPID. Ele é considerado como um conceito que viabiliza a articulação entre as duas zonas (eixos organizadores e interface proposicional interdisciplinar) apresentadas no MC.

\section{Resultados e discussão}

O mapeamento conceitual dos conteúdos programáticos das disciplinas $\mathrm{CN}$ e PET resultou na elaboração de $2 \mathrm{MCs}$ contendo 30-40 conceitos cada. Eles representaram a forma pela qual a aluna percebeu essas disciplinas gerais do Ciclo Básico. Na sequência, os docentes P.R.M.C. e P.J.G. se reuniram com a aluna G.B.C. e revisaram os MCs de CN e PET, respectivamente. Duas sessões de uma hora foram necessárias para combinar a representação da aula com o olhar do docente em cada caso. O resultado final ainda foram MCs grandes (cerca 
Figura 4. MC-5 relacionando os conceitos das disciplinas A e B. A interface proposicional interdisciplinar (IPID) está destacada por um retângulo tracejado (C5-C8) e por conceitos em caixas pretas. Estes conceitos podem revelar conexões latentes entre os conteúdos disciplinares.

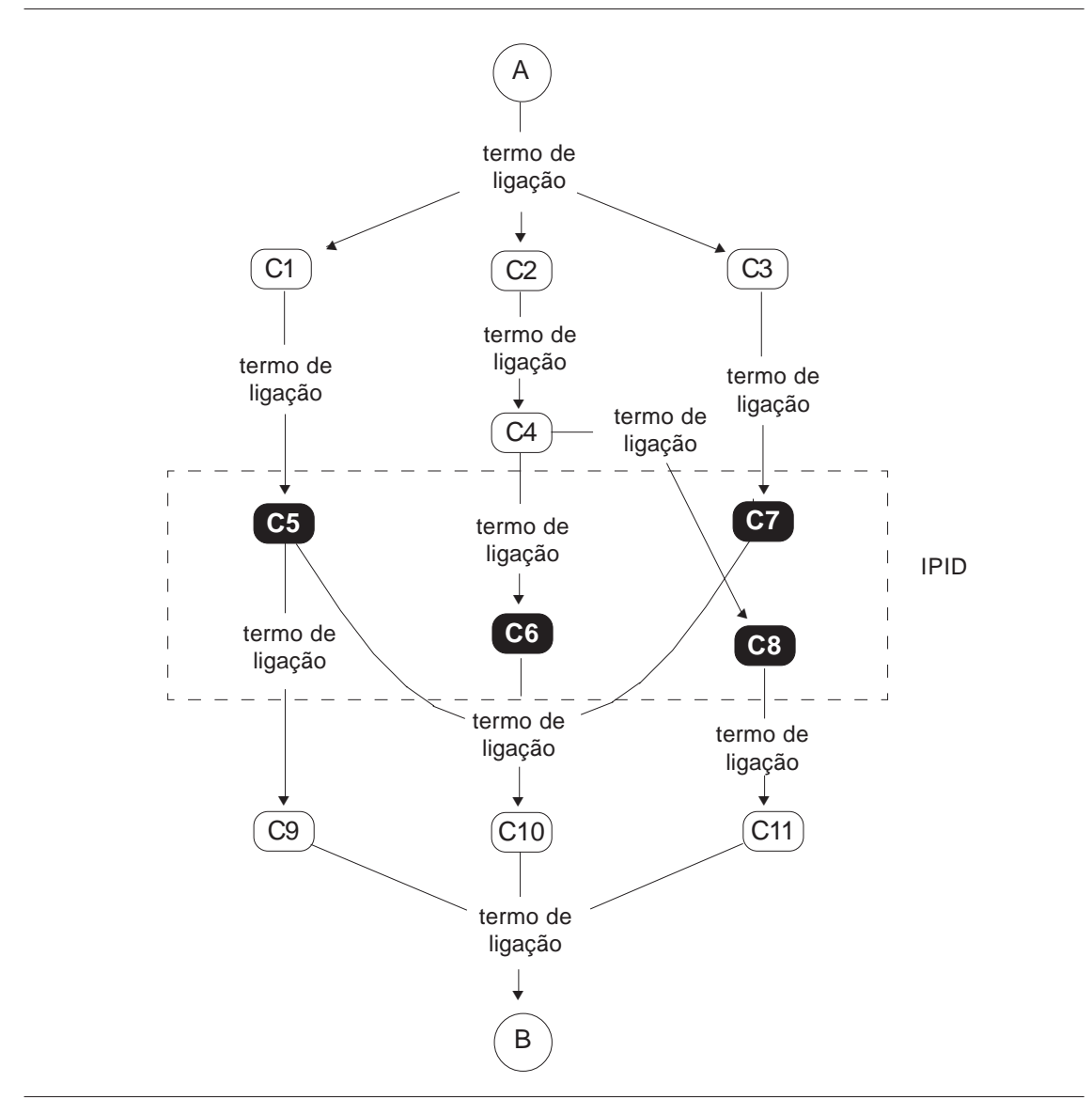

Fonte: Elaborada pelos autores

de trinta conceitos), mas com uma hierarquia melhor organizada: a visão do docente responsável pela disciplina permitiu organizar os conceitos em função do seu nível abrangência. Desta forma, esses MCs apresentaram os conceitos mais abrangentes (organizadores das disciplinas) como pontos de partida para a leitura das proposições. A busca por conceitos promissores para a promoção de vínculos entre as disciplinas CN e PET foi desenvolvida em uma entrevista reunindo todos os envolvidos, durante quatro horas.

Os MCs 1, 2, 3 e 4 de CN e PET foram sendo modificados a cada etapa. As etapas de 1 a 4 foram fundamentais para a etapa 5, pois os MC-4 das disciplinas A e B foram apresentados, aos docentes responsáveis, em uma entrevista. A leitura conjunta dos MC-4 na entrevista resultou no estabelecimento de relações entre as disciplinas A e B. Os responsáveis pelas disciplinas, juntamente com a aluna e a especialista em MCs, conseguiram visualizar, de forma clara, os temas que são possíveis conectores entre os conteúdos das disciplinas envolvidas. Esses temas foram representados por conceitos, os quais foram utilizados para criar a 
interface proposicional interdisciplinar (IDPI) entre as disciplinas CN e PET. O MC final deste processo (MC-5) é apresentado na Figura 5.

Figura 5. MC-5 relacionando os conceitos da disciplina CN e PET. A interface proposicional interdisciplinar (IPID) está destacada com conceitos em caixas retangulares pretas (tecnologia, complexidade e religião). Ela contém os mais promissores vínculos conceituais entre os conteúdos programáticos das disciplinas.

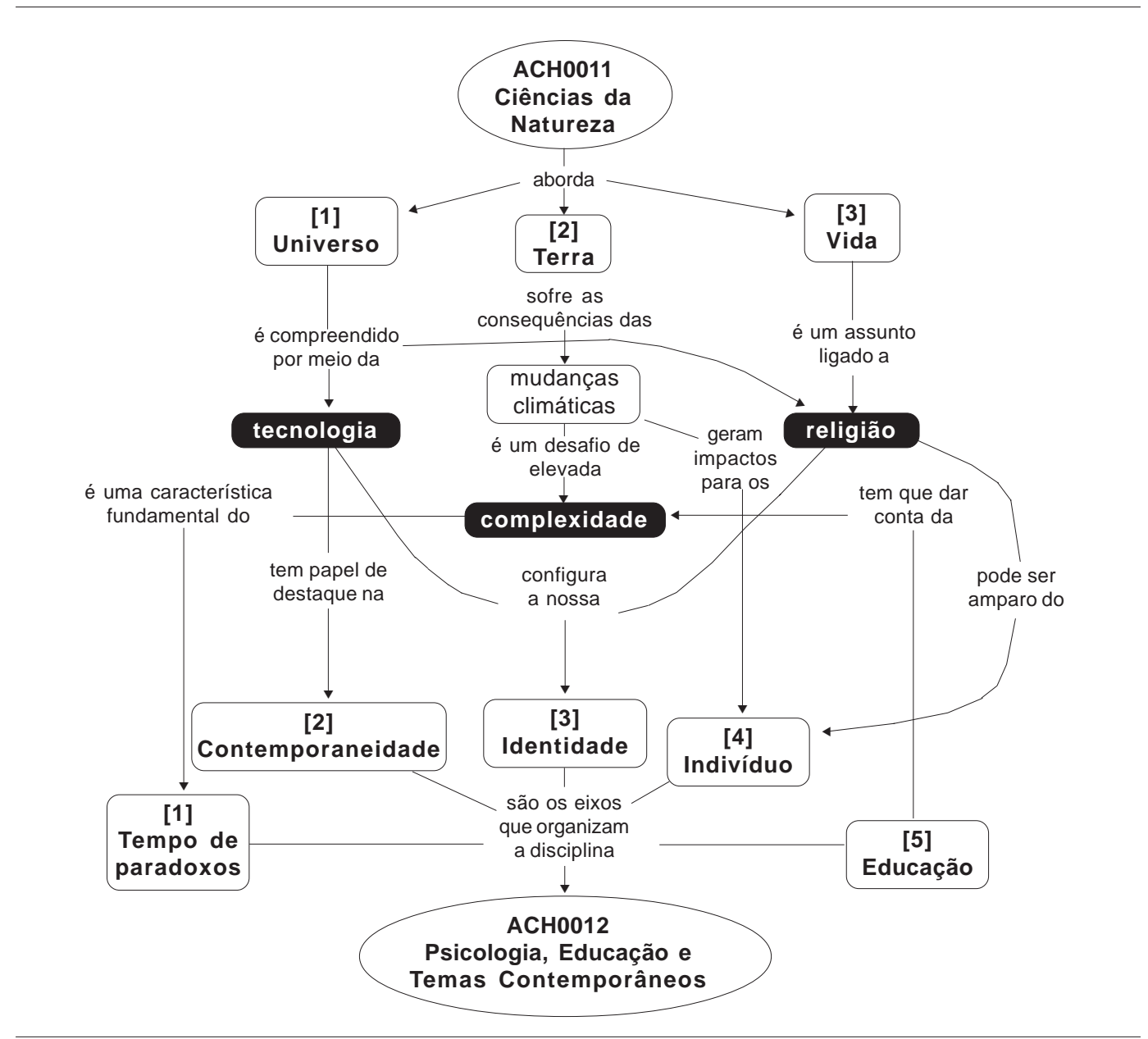

Fonte: Elaborada pelos autores

A leitura do $\mathrm{MC}$ começa pela disciplina $\mathrm{CN}$ já que ela é ministrada antes da disciplina PET (Quadro 1). Os conceitos "universo", “Terra", e "vida” são os eixos organizadores de CN que ajudaram a identificar possíveis vinculações com o conteúdo programático de PET. Para isso, foram considerados também os conceitos que representam os eixos organizadores de PET, que são "tempo de paradoxo", "contemporaneidade", "identidade", "indivíduo" e "educação". Com a interface entre as disciplinas, foi possível identificar os conceitos "tecnologia", "complexidade" e "religião" como os mais promissores vínculos conceituais entre os conteúdos programáticos das disciplinas. 
O conceito "tecnologia" (Figura 5) permite o acoplamento das disciplinas CN e PET, quando se consideram os eixos temáticos "universo" (CN), "contemporaneidade" (PET) e "identidade" (PET). A tecnologia possui vinculações com a ciência (universo "é compreendido por meio da $\rightarrow$ tecnologia), além de estar intrinsecamente relacionada com a contemporaneidade (tecnologia " tem papel de destaque na $\rightarrow$ contemporaneidade). O panorama do século XXI evidencia o rápido crescimento científico e tecnológico, os quais produzem consequências na identidade dos indivíduos que vivem na sociedade do conhecimento (tecnologia " configura nossa $\rightarrow$ identidade). Este novo cenário é um desafio a ser enfrentado no sentido de formar cidadãos capazes de compreenderem os fenômenos complexos.

A "complexidade" (Figura 5) é um conceito-chave nesse processo de aproximação interdisciplinar, pois permite: descrever a sociedade atual e fomentar o diálogo disciplinar (diálogo entre as culturas da área de exatas e biológicas com humanas). A sociedade atual exige uma abordagem da educação que proporcione condições para os cidadãos adquirirem habilidades para resolução de problemas complexos (FORD; FORMAN, 2006; MORAN, 2002), tal como expressa a proposição "educação " tem que dar conta da $\rightarrow$ complexidade". O restabelecimento do diálogo entre as duas culturas, caracterizadas por C. P. Snow (1995), é fundamental para construir um entendimento preciso sobre temas complexos como as mudanças climáticas (mudanças climáticas "é um desafio de elevada $\rightarrow$ complexidade). A compreensão das mudanças climáticas pode desempenhar papel fundamental, visto que ela integra os avanços científico-tecnológicos (elementos da cultura da área de exatas e biológicas) provenientes da disciplina $\mathrm{CN}$ e os seus desdobramentos para os indivíduos (elementos da cultura da área de humanas) oriundos da disciplina PET, fomentando o diálogo disciplinar.

O conceito "religião" (Figura 5) permite o acoplamento das disciplinas CN e PET, quando se consideram os eixos temáticos "vida" (vida " é um assunto ligado a $\rightarrow$ religião) e "indivíduo" (religião - pode ser amparo do $\rightarrow$ indivíduo). As discussões sobre o conceito "vida" nos permitem remeter aos avanços recentes da biologia molecular e seus desdobramentos éticos, já que este assunto contempla as crenças dos "indivíduos" e suas concepções sobre a "religião". Perspectivas complementares sobre o papel da religião no desenvolvimento da sociedade (religião " configura nossa $\rightarrow$ identidade) e da ciência (universo "é compreendido por meio da $\rightarrow$ religião) são caminhos promissores para aproximar as disciplinas mapeadas.

Os conceitos e as proposições apresentadas no IPID oferecem um ponto de partida importante para os professores pensarem em ações concretas em termos de métodos de ensino e materiais didáticos. Identificar estes conceitos (IPID) é apenas uma parte do caminho para conectar melhor as disciplinas. O passo seguinte explora a informação obtida usando MCs para fazer a interdisciplinaridade acontecer na sala de aula. O objetivo final é fazer os alunos perceberem a relevância de conectar o conhecimento disciplinar para entender os problemas de alta complexidade do século XXI (verificar esta frase: redação confusa).

\section{Considerações finais}

Os MCs são ferramentas gráficas de representação da informação e do conhecimento úteis para processos colaborativos. Essa é uma condição característica de contextos interdisciplinares, onde vários saberes precisam ser compreendidos, compartilhados e negociados 
por todos. O presente trabalho explorou o mapeamento conceitual para identificar pontos de acoplamento entre as disciplinas Ciências da Natureza e Psicologia, Educação e Temas Contemporâneos. Após quatro etapas de elaboração, revisão e análise dos MCs, foi possível representar a interface entre as disciplinas e identificar os conceitos "tecnologia", "complexidade" e "religião" como os promissores para estimular a interdisciplinaridade.

O uso de MCs para estimular a integração curricular tem grande potencial para identificação de relações interdisciplinares, visto a grande dificuldade de estabelecimento de vínculos entre os conteúdos disciplinares. A utilização dos procedimentos descritos neste trabalho pode ser útil para que docentes verifiquem a existência (ou não) de vínculos conceituais entre os conteúdos programáticos de suas disciplinas. Certamente, há espaço na literatura para explorar a existência de integrações disciplinares usando MCs em outros contextos educacionais, a fim de verificar a robustez das conclusões obtidas consideradas nessa oportunidade.

\section{Agradecimentos}

Os autores agradecem às agências de fomento à pesquisa que financiam os trabalhos desenvolvidos pelo nosso grupo de pesquisa (CNPq-553710/2006-0, 486194/2011-6; CAPES-3555-09-7; FAPESP-2006/ 03083-0, 2008/04709-6, 2011/09941-7).

\section{Referências}

BAKER, M. et al. The role of grounding incollaborative learning tasks. In: DILLENBOURG, P. (Ed). Collaborative learning: cognitive and computational approaches. Oxford: Pergamon, 1999.

BAKER, V. L.; LATTUCA, L. R. Developmental networks and learning: toward an interdisciplinary perspective on identity development during doctoral study. Studies in Higher Education, Oxfordshire, v. 35, n.7, p. 807-827, 2010.

CORREIA, P. R. M. The use of concept maps for knowledge management: from classrooms to research labs. Analytical and Bioanalytical Chemistry, Heidelberg, v. 402, n. 6, p. 1979-1986, 2012.

CORREIA, P. R. M. et al. The importance of scientific literacy in fostering education for sustainability: Theoretical considerations and preliminary findings from a Brazilian experience. Journal of Cleaner Production, Oxford, v. 18, n. 7, p. 678-85, 2010.

CORREIA, P. R. M.; INFANTE-MALACHIAS, M. E.; GODOY, C. E. C. Proceedings of the Third International Conference on Concept Mapping, Tallin, Estonia; Helsinki, Finlândia: OÜ Vali Press, 2008.

CRANDALL, B.; KLEIN, G.; HOFFMAN, R. R. Working minds: a practitioner's guide to cognitive task analysis. Cambridge: MIT Press, 2006.

DAVIES, M. Concept mapping, mind mapping and argument mapping: what are the differences and do they matter? Higher Education, Oxford, v. 62, n. 3, p. 279-301, 2011.

FISCHER, F. et al. Fostering collaborative knowledge construction with visualization tools. Learning and Instruction, Oxford, v. 12, n. 2, p. 213-232, 2002. 
FORD, M. J.; FORMAN, E. A. Chapter 1: Redefining Disciplinary Learning in Classroom Contexts. Review of Research in Education, Thousand Oaks, v. 30, n. 1, p. 1-32, 2006.

GOMES, C. B. USP Leste: a expansão da universidade do oeste para leste. São Paulo: Edusp, 2005.

KLEIN, J. T. Crossing boundaries: knowledge, disciplinarities and interdisciplinarities. Charlottesville: University Press of Virginia, 1996.

LAMBERT, A. D.; TERENZINI, P. T.; LATTUCA, L. R. More than meets the eye: curricular and programmatic effects on student learning. Research in Higher Education, New York, v. 48, n. 2 , p.141-168, 2007.

LATTUCA, L. R. Learning interdisciplinarity: sociocultural perspectives on academic work. Journal of Higher Education, Columbus, v. 73, n. 6, p.711-739, 2002.

Creating interdisciplinarity: interdisciplinary research and teaching among college and university faculty. Nashville: Vanderbilt University Press, 2001.

LATTUCA, L. R.; STARK, J. S. Will disciplinary perspectives impede curricular reform. Journal of Higher Education, Columbus, v. 65, n. 4, p. 401-426, 1994.

LATTUCA, L. R.; VOIGT, L. J.; FATH, K. Q. Does interdisciplinarity promote learning? Theoretical support and researchable questions. The Review of Higher Education, v. 28, n. 1, p. 23-48, 2004.

MOON, B. et al. Applied concept mapping: capturing, analyzing, and organizing knowledge. Boca Raton: CRC Press, 2011.

MORAN, J. Interdisciplinarity: the new critical idiom. London: Routledge, 2002.

NOVAK, J. D. Meaningful learning: the essential factor for conceptual change in limited or inappropriate propositional hierarchies leading to empowerment of learners. Science Education, Hoboken, v. 86, n. 4, p. 548-571, 2002.

. Learning, creating, and using knowledge: concept maps as facilitative tools in schools and corporations. New York: Routledge, 2010.

SAWYER, R. K. Educating for innovation. Thinking Skills and Creativity, Oxford, v. 1, n. 1, p. 41-48, 2006.

SNOW, C. P. As duas culturas e uma segunda leitura. São Paulo: Edusp, 1995.

STARK, J. S. et al. Program-level curriculum planning: an exploration of faculty perspectives on two different campuses. Research in Higher Education, New York, v. 38, n. 1, p. 99-130, 1997.

TORRES, P. L.; MARRIOTTT, R. C. V. Handbook of research on collaborative learning using concept mapping. Hershey: IGI Global, 2009.

UNESCO. Towards knowledge societies: UNESCO world report. Paris: Unesco Publishing, 2005. WEINGART, P.; STEHR, N. Practising interdisciplinarity. Toronto: University of Toronto Press, 2000. 